\title{
Editorial
}

\section{Acknowledgement to Reviewers of Antibiotics in 2013}

\section{Antibiotics Editorial Office}

MDPI AG, Klybeckstrasse 64, CH-4057 Basel, Switzerland

Published: 27 February 2014

The editors of Antibiotics would like to express their sincere gratitude to the following reviewers for assessing manuscripts in 2013:

\begin{tabular}{lll} 
Alfonzo, Antonio & Gálvez, Antonio & Mueller, Eric W \\
Álvarez-Ordóñez, A. & Hall, Tony J. & Nielsen, Kaare M. \\
Asuri, Prashanth & Ho, Pak-Leung & Nielsen-Preiss, Sheila M. \\
Bettina, Genthe & Hughes, Diarmaid & Oostenbrink, R \\
Björklund, Erland & Hung, Deborah T. & Opperman, Timothy J. \\
Bohnert, Jürgen A. & Huttner, Benedikt & Pakhomova, Svetlana \\
Calokerinos, Anthony & Jungnickel, Christian & Partridge, Sally R. \\
Chachaty, Elisabeth & Kaatz, Glenn W. & Perez, Astrid \\
Chang, Feng-Yee & Kiffer, Carlos & Pérez, Lourdes \\
Charani, Esmita & Kirst, Herbert A & Pletz, Mathias \\
Chen, Zhe-Sheng & Klitgaard, Janne & Pridmore, Andrew \\
Cheng, V. C. C. & Koutchma, Tatiana & Pruden, Amy \\
Chou, Chi-chung & Krapac, Ivan G. & Qin, Zhiqiang \\
Cody, Vivian & Leone, Marc & Rineh, Ardeshir \\
Collins, James & Lesprit, Philippe & Rodriguez-Meizoso, Irene \\
Costelloe, Ceire & Lin, Yu-Hsin & Roemer, Terry \\
Courvalin, Patrice & Llor, Carles & Roque, Fátima \\
Demirjian, Alicia & Lous, Jørgen & Samaras, Vasilios \\
Doron, Shira & Mañes, Jordi & Samarasundera, Edgar \\
Dreier, Jürg & Martinez, Luis R. & Sass, Peter \\
Drlica, Karl & Mazel, Diddier & Schweizer, Herbert \\
Fatta-Kassinos, D. & Mcintosh, E. David G. & Shigemura, Katsumi \\
Fralick, Joe A. & Moir, Donald T & Simpson, Jamie \\
\hline
\end{tabular}


Siqueira-Junior, José P.

Slain, Douglas

Solich, Petr

Solichova, Dagmar

Symmons, Martyn F.

Tatavarthy, Aparna

Theuretzbacher, Ursula
Thorsen, Line

Thulesius, Hans

Tiwari, Amit

Topp, Edward

Tsai, J.C.

Turner, R. Brigg

Van Der Velden, Alike
Victor, T. C.

White, Steve

Wood, Fiona

Woodward, Martin

Wu, Jiunn-Jong

(C) 2014 by the authors; licensee MDPI, Basel, Switzerland. This article is an open access article distributed under the terms and conditions of the Creative Commons Attribution license (http://creativecommons.org/licenses/by/3.0/). 